九州大学学術情報リポジトリ

Kyushu University Institutional Repository

\title{
Stable Matchings with Ties, Master Preference Lists, and Matroid Constraints
}

Kami yama, Naoyuki

Institute of Mathematics for Industry, Kyushu University | JST, PRESTO

http://hdl. handle. net/2324/1500418

出版情報 : Lecture Notes in Computer Science. 9347, pp.3-14, 2015. Springer Berlin Heidelberg バージョン：

権利関係 : 


\section{Preprint Series}

Mathematics for Industry

Kyushu University

\section{Stable Matchings with Ties, Master Preference Lists, and Matroid Constraints}

\section{Naoyuki Kamiyama}

\section{2015-3}

( Received April 23, 2015 )

Institute of Mathematics for Industry

Graduate School of Mathematics

Kyushu University

Fukuoka, JAPAN 


\title{
Stable Matchings with Ties, Master Preference Lists, and Matroid Constraints
}

\author{
Naoyuki Kamiyama*†‡
}

\begin{abstract}
In this paper, we consider a matroid generalization of the hospitals/residents problem with ties and master lists. In this model, the capacity constraints for hospitals are generalized to matroid constraints. By generalizing the algorithms of O'Malley for the hospitals/residents problem with ties and master lists, we give polynomial-time algorithms for deciding whether there exist a super-stable matching and a strongly stable matching in our model, and finding such matchings, if they exist.
\end{abstract}

\section{Introduction}

The stable matching problem introduced by Gale and Shapley [4] is one of the most popular mathematical models of a matching problem in which agents have preferences. It is known [4] that if preference lists do not contain ties, then there always exists a stable matching and we can find one in polynomial time. However, if there exist ties in preference lists, then the situation dramatically changes. In the stable matching problem with ties, three stability concepts were proposed by Irving [5] (see also Chapter 3 of [11] for a survey of the stable matching problem with ties). The first one is called the weak stability. This stability concept guarantees that there exists no unmatched pair each of whom prefers the other to the current partner. It is known [5] that there always exists a weakly stable matching and we can find one in polynomial time by slightly modifying the algorithm of [4]. The second one is called the strong stability. This stability concept guarantees that there exists no unmatched pair such that (i) there exists an agent $a$ in this pair that prefers the other to the current partner, and (ii) the agent in this pair other than $a$ prefers $a$ to the current partner, or is indifferent between $a$ and the current partner. The last one is called the super-stability. This stability concept guarantees that there exists no unmatched pair each of whom prefers the other to the current partner or is indifferent between the other and the current partner.

One of the most notable differences between the last two concepts and the stability concept in the stable matching problem without ties is that there may exist no stable matching [5]. Thus, from the algorithmic viewpoint, it is important to reveal whether we can check the existence of matchings satisfying such stability concepts in polynomial time. For the one-to-one setting (i.e., the stable matching problem with ties), Irving [5] gave polynomial-time algorithms for finding a super-stable matching and a strongly stable matching (see also [10]). For the manyto-one setting (i.e., the hospitals/residents problem with ties), Irving, Manlove, and Scott [6] gave a polynomial-time algorithm for finding a super-stable matching, and Irving, Manlove, and

\footnotetext{
${ }^{*}$ Institute of Mathematics for Industry, Kyushu University, Fukuoka, Japan

${ }^{\dagger}$ JST, PRESTO, Saitama, Japan

${ }^{\ddagger}$ kamiyama@imi.kyushu-u.ac.jp
} 
Scott [7] a polynomial-time algorithm for finding a strongly stable matching. It should be noted that Kavitha, Mehlhorn, Michail, and Paluch [9] proposed a faster algorithm for this setting.

In this paper, we consider a matroid generalization of the hospitals/residents problem with ties. In our model, the capacity constraints for hospitals are generalized to matroid constraints. In contrast to the stable matching problem without ties (and the weak stability) [3], to the best of our knowledge, it is open whether we can extend the results about the super-stability and the strong stability to the matroid setting. In this paper, as a step toward the settlement of this question, we focus on the situation in which we are given a master list and the preference list of each hospital over residents is derived from this master list. For the stable matching problem with ties and master lists, Irving, Manlove, and Scott [8] gave simple polynomial-time algorithms for finding a super-stable matching and a strongly stable matching. Furthermore, O'Malley [12] gave polynomial-time algorithms for finding a super-stable matching and a strongly stable matching in the hospitals/residents problem with ties and master lists. In this paper, by generalizing the algorithms of [12], we give polynomial-time algorithms for finding a super-stability matching and a strongly stable matching in a matroid generalization of the hospitals/residents problem with ties and master lists, i.e., a partial positive answer for the above question.

It should be noted that Abraham, Irving, and Manlove [1] posed an open problem related to the super-stability and the strongly stability in the student-project allocation problem that is a variation of the hospitals/residents problem having hierarchical capacity constraints (see also [11, p.268]). As pointed in [1], these hierarchical capacity constraints can be expressed by using a matroid. However, since the definitions of the super-stability and the strong stability in the student-project allocation problem with ties were not explicitly given in [1], we do not discuss about the relationship between their model and our model. Instead, we give a concrete example in the student-project allocation setting that our model can express.

The rest of this paper is organized as follows. In Section 2, we formally define our problem and give basics of matroids. In Sections 3 and 4, we give polynomial-time algorithms for finding a super-stable matching and a strongly stable matching in our model, respectively. In Section 5, we give examples of concrete models that our model can express.

\section{Preliminaries}

For each set $X$ and each element $x$, we define $X+x:=X \cup\{x\}$ and $X-x:=X \backslash\{x\}$. A pair $\mathbf{M}=(U, \mathcal{I})$ is called a matroid, if $U$ is a finite set and $\mathcal{I}$ is a family of subsets of $U$ satisfying the following conditions.

(I0) $\emptyset \in \mathcal{I}$.

(I1) If $I \in \mathcal{I}$ and $J \subseteq I$, then $J \in \mathcal{I}$.

(I2) If $I, J \in \mathcal{I}$ and $|I|<|J|$, then $I+u \in \mathcal{I}$ for some element $u$ in $J \backslash I$.

\subsection{Problem formulation}

In this paper, we are given a simple bipartite graph $G=(R \cup H, E)$ such that its vertex set is partitioned into two disjoint subsets $R$ and $H$, and every edge in $E$ connects a vertex in $R$ and a vertex in $H$. We call a vertex in $R$ (resp., $H$ ) a resident (resp., a hospital). For each vertex $v$ in $R \cup H$ and each subset $F$ of $E$, we denote by $F(v)$ the set of edges in $F$ incident to $v$. For each resident $r$ in $R$ and each hospital $h$ in $H$, if there exists an edge in $E$ connecting $r$ and $h$, then we denote by $(r, h)$ this edge. Furthermore, we are given a matroid $\mathbf{N}=(E, \mathcal{F})$ such that 
$\{e\} \in \mathcal{F}$ for every edge $e$ in $E$. The matroid $\mathbf{N}$ represents constraints on assignment of residents to hospitals. For example, it can express capacity constraints in the hospitals/residents problem (see Section 5). For each resident $r$ in $R$, we are given a reflexive and transitive binary relation $\succsim_{r}$ on $E(r) \cup\{\emptyset\}$ such that at least one of $e \succsim_{r} f$ and $f \succsim_{r} e$ holds for every edges $e, f$ in $E(r)$, and $e \succsim_{r} \emptyset$ and $\emptyset \succsim_{r} e$ for every edge $e$ in $E(r)$. For each resident $r$ in $R$, the binary relation $\succsim_{r}$ represents the preference list of $r$. Namely, for each resident $r$ in $R$ and each edges $e, f$ in $E(r), e \succsim_{r} f$ means that $r$ preferes $e$ to $f$, or is indifferent between $e$ and $f$. For each resident $r$ in $R$ and each edges $e, f$ in $E(r)$, we write $e \succ_{r} f$ (resp., $e \sim_{r} f$ ), if $e \succsim_{r} f$ and $e \succsim_{r} f$ (resp., $e \succsim_{r} f$ and $\left.f \succsim_{r} e\right)$. Furthermore, we are given a reflexive and transitive binary relation $\succsim_{H}$ on $R$ such that at least one of $r \succsim_{H} s$ and $s \succsim_{H} r$ holds for every residents $r, s$ in $R$. The binary relation $\succsim_{H}$ represents the master preference list of hospitals in $H$. We define the notations $\succ_{H}$ and $\sim_{H}$ in the same way as $\succ_{r}$ and $\sim_{r}$ for a resident $r$ in $R$.

A subset $M$ of $E$ is called a matching in $G$, if

(M1) $|M(r)| \leq 1$ for every resident $r$ in $R$, and

(M2) $M \in \mathcal{F}$.

For each matching $M$ in $G$ and each resident $r$ in $R$ such that $M(r) \neq \emptyset$, we do not distinguish between $M(r)$ and its unique element. For each matching $M$ in $G$ and each edge $e=(r, h)$ in $E \backslash M$, we say that $r$ weakly (resp., strictly) prefers $e$ on $M$, if $e \succsim_{r} M(r)$ (resp., $e \succ_{r} M(r)$ ). In addition, for each matching $M$ in $G$ and each edge $e=(r, h)$ in $E \backslash M$, we say that $H$ weakly (resp., strictly) prefers e on $M$, if

(P1) $M+e \in \mathcal{F}$, and/or

(P2) there exists an edge $f=(s, p)$ in $M$ such that $M+e-f \in \mathcal{F}$ and $r \succsim_{H} s$ (resp., $r \succ_{H} s$ ).

A matching $M$ in $G$ is said to be super-stable, if there exists no edge $(r, h)$ in $E \backslash M$ such that $r$ and $H$ weakly prefer $(r, h)$ on $M$. Furthermore, a matching $M$ in $G$ is said to be strongly stable, if there exists no edge $(r, h)$ in $E \backslash M$ such that

(S1) $r$ and $H$ weakly prefer $(r, h)$ on $M$, and

(S2) at least one of $r$ and $H$ strictly prefers $(r, h)$ on $M$.

Unfortunately, it is known [5] that there may exist no matching satisfying the above conditions. Thus, our goal is to give a polynomial-time algorithm for deciding whether there exists a matching satisfying the above conditions, and find such a matching, if one exists.

\subsection{Basics of matroids}

Let $\mathbf{M}=(U, \mathcal{I})$ be a matroid. A subset of $U$ belonging to $\mathcal{I}$ is called an independent set of $\mathbf{M}$. A subset $C$ of $U$ is called a circuit of $\mathbf{M}$, if $C$ is not an independent set of $\mathbf{M}$, but every proper subset of $C$ is an independent set of $\mathbf{M}$. Notice that the condition (I1) implies that for every subset $X$ of $U$, if there exists a circuit of $\mathbf{M}$ that is a subset of $X$, then $X \notin \mathcal{I}$. Let $I$ and $u$ be an independent set of $\mathbf{M}$ and an element in $U \backslash I$ such that $I+u \notin \mathcal{I}$, respectively. It is known [13, Proposition 1.1.6] that there exists a unique circuit of $\mathbf{M}$ that is a subset of $I+u$, and $u$ belongs to this unique circuit. This circuit is called the fundamental circuit of $u$, and denoted by $\mathrm{C}_{\mathbf{M}}(u, I)$. Define

$$
\mathrm{C}_{\mathbf{M}}^{-}(u, I):=\mathrm{C}_{\mathbf{M}}(u, I)-u .
$$


It is known [13, p.20, Exercise 5] that we have

$$
\mathrm{C}_{\mathbf{M}}(u, I)=\{w \in I+u \mid I+u-w \in \mathcal{I}\} .
$$

It should be noted that for each subset $M$ of $E$ and each edge $e$ in $E \backslash M$ such that $M+e \notin \mathcal{F}$, the condition (P2) can be restated as follows.

- There exists an edge $f=(s, p)$ in $\mathrm{C}_{\mathbf{N}}^{-}(e, M)$ such that $r \succsim_{H} s$ (resp., $r \succ_{H} s$ ).

A maximal independent set of $\mathbf{M}$ is called a base of $\mathbf{M}$. The condition (I2) implies that every base of $\mathbf{M}$ has the same size. For each subset $X$ of $U$, we define

$$
\mathcal{I}|X:=\{J \subseteq X \mid J \in \mathcal{I}\}, \quad \mathbf{M}| X:=(X, \mathcal{I} \mid X) .
$$

It is known [13, p.20] that for each subset $X$ of $U, \mathbf{M} \mid X$ is a matroid. For each subset $X$ of $U$, we define $\mathbf{r}_{\mathbf{M}}(X)$ as the size of a base of $\mathbf{M} \mid X$. It is not difficult to see that for every subset $B$ of $U, B$ is a base of $\mathbf{M}$ if and only if $|B|=\mathrm{r}_{\mathbf{M}}(U)$. For each disjoint subsets $X, J$ of $U$, we define

$$
\mathrm{p}(J ; X):=\mathrm{r}_{\mathbf{M}}(J \cup X)-\mathrm{r}_{\mathbf{M}}(X) .
$$

For each subset $X$ of $U$, we define

$$
\mathcal{I} / X:=\{J \subseteq U \backslash X|\mathrm{p}(J ; X)=| J \mid\}, \quad \mathbf{M} / X:=(U \backslash X, \mathcal{I} / X) .
$$

It is known [13, Proposition 3.1.6] that for each subset $X$ of $U, \mathbf{M} / X$ is a matroid.

The following property of circuits is used in the sequel.

Theorem 1 (See, e.g., [13, p.15, Exercise 14]). Assume that we are given a matroid $\mathbf{M}=(U, \mathcal{I})$ and circuits $C_{1}, C_{2}$ of $\mathbf{M}$ such that $C_{1} \cap C_{2} \neq \emptyset$ and $C_{1} \backslash C_{2} \neq \emptyset$. Then, for every element $u$ in $C_{1} \cap C_{2}$ and every element $w$ in $C_{1} \backslash C_{2}$, there exists a circuit $C$ of $\mathbf{M}$ such that $w \in C$ and $C$ is a subset of $\left(C_{1} \cup C_{2}\right)-u$.

Although the following lemmas easily follow, we give their proofs for completeness.

Lemma 2. Assume that we are given a matroid $\mathbf{M}=(U, \mathcal{I})$ and independent sets $I, J$ of $\mathbf{M}$ such that $I \subseteq J$. Then, for every element $u$ in $U \backslash J$ such that $I+u \notin \mathcal{I}$, we have $J+u \notin \mathcal{I}$ and $\mathrm{C}_{\mathrm{M}}(u, I)=\mathrm{C}_{\mathrm{M}}(u, J)$.

Proof. Let $u$ be an element in $U \backslash J$ such that $I+u \notin \mathcal{I}$. Since $I \subseteq J, \mathrm{C}_{\mathbf{M}}(u, I)$ is a subset of $J+u$, which implies that $J+u \notin \mathcal{I}$. Define $C_{1}:=\mathrm{C}_{\mathbf{M}}(u, I)$ and $C_{2}:=\mathrm{C}_{\mathbf{M}}(u, J)$. Assume that $C_{1} \neq C_{2}$. Since $u$ belongs to $C_{1} \cap C_{2}$, Theorem 1 implies that there exists a circuit $C$ of $\mathbf{M}$ such that $C$ is a subset of $\left(C_{1} \cup C_{2}\right)-u$. In addition, since $C_{1}-u \subseteq I \subseteq J$ and $C_{2}-u \subseteq J$, we have $C \subseteq J$. This contradicts the fact that $J \in \mathcal{I}$.

Lemma 3. Assume that we are given a matroid $\mathbf{M}=(U, \mathcal{I})$, circuits $C, C_{1}, C_{2}, \ldots, C_{k}$ of $\mathbf{M}$, and elements $u_{1}, u_{2}, \ldots, u_{k}$ in $U$ such that $u_{i} \in C \cap C_{i}$ for every $i=1,2, \ldots, k$. In addition, we assume that there exists an element $w$ in $U$ such that $w \in C$ and $w \notin C_{i}$ for every $i=1,2, \ldots, k$. Then, there exists a circuit $C^{\prime}$ of $\mathbf{N}$ such that $C^{\prime} \subseteq\left(C \cup C_{1} \cup C_{2} \cup \cdots \cup C_{k}\right) \backslash\left\{u_{1}, u_{2}, \ldots, u_{k}\right\}$.

Proof. We consider the following procedure.

Step 1. Set $t:=1$ and $K_{0}:=C$.

Step 2. If $t \leq k$, then do the following steps. Otherwise, go to Step 3. 
(2-a) If $u_{t} \notin K_{t-1}$, then go to Step (2-c).

(2-b) Find a circuit $K_{t}$ of $\mathbf{N}$ such that $w \in K_{t}$ and $K_{t}$ is a subset of $\left(K_{t-1} \cup C_{t}\right)-u_{t}$.

(2-c) Update $t:=t+1$ and go back to the beginning of Step 2.

Step 3. Output $K_{k}$ and halt.

Notice that Theorem 1 implies that in Step (2-b) there exists a circuit $K_{t}$ satisfying the above properties. It is not difficult to see that $K_{k}$ is a subset of $C \cup C_{1} \cup C_{2} \cup \cdots \cup C_{k}$ and $u_{i} \notin K_{k}$ for every $i=1,2, \ldots, k$. This completes the proof.

Lemma 4. Assume that we are given a matroid $\mathbf{M}=(U, \mathcal{I})$ and a subset $X$ of $U$.

1. Let $B$ be an arbitrary base of $\mathbf{M} \mid X$. For every subset $I$ of $U \backslash X, I$ is an independent set of $\mathbf{M} / X$ if and only if $I \cup B$ is an independent set of $\mathbf{M}$.

2. For every base $B_{1}$ of $\mathbf{M} \mid X$ and every base $B_{2}$ of $\mathbf{M} / X, B_{1} \cup B_{2}$ is a base of $\mathbf{M}$.

3. For every subset $I$ of $U$ such that $I \cap X$ is a base of $\mathbf{M} \mid X$, if $I \backslash X$ is not an independent set of $\mathbf{M} / X$, then $I$ is not an independent set of $\mathbf{M}$.

Proof. The statement (1) follows from Proposition 3.1.7 of [13]. Next we consider the statement (2). Assume that $B_{1} \cup B_{2}$ is not a base of $\mathbf{M}$. Then, there exists an element $u$ in $U \backslash\left(B_{1} \cup B_{2}\right)$ such that $\left(B_{1} \cup B_{2}\right)+u$ is an independent set of $\mathbf{M}$. If $u$ belongs to $X$, then this contradicts the fact that $B_{1}$ is a base of $\mathbf{M} \mid X$. Thus, $u$ does not belong to $X$. Since $B_{1}$ is a base of $\mathbf{M}$, the statement (1) implies that $B_{2}+u$ is an independent set of $\mathbf{M} / X$. This contradicts the fact that $B_{2}$ is a base of $\mathbf{M} / X$. Lastly we prove the statement (3). Assume that $I \in \mathcal{I}$. Since $I \cap X$ is a base of $\mathbf{M} \mid X$, the statement (1) implies that $I \backslash X$ is an independent set of $\mathbf{M} / X$. This contradicts the fact that $I \backslash X$ is not an independent set of $\mathbf{M} / X$.

Let $\mathbf{M}_{1}=\left(U, \mathcal{I}_{1}\right)$ and $\mathbf{M}_{2}=\left(U, \mathcal{I}_{2}\right)$ be matroids on the same ground set. A subset $I$ of $U$ is called a common independent set of $\mathbf{M}_{1}$ and $\mathbf{M}_{2}$, if $I$ belongs to $\mathcal{I}_{1} \cap \mathcal{I}_{2}$. It is known that we can find a maximum-size common independent set in time bounded by a polynomial in $|U|$ and $\gamma$, where $\gamma$ is the time required to decide whether $X$ is a common independent set of $\mathbf{M}_{1}$ and $\mathbf{M}_{2}$ for one subset $X$ of $U$. If we use the algorithm proposed by Cunningham [2], then we can find a maximum-size common independent set in $O\left(|U|^{2.5} \gamma\right)$ time.

\section{Super-Stable Matchings}

In this section, we consider the problem of deciding whether there exists a super-stable matching in $G$, and find such a matching, if one exists. We first partition $R$ into non-empty disjoint subsets $R_{1}, R_{2}, \ldots, R_{n}$ such that

1. $r \sim_{H} s$ for every $i=1,2, \ldots, n$ and every residents $r, s$ in $R_{i}$, and

2. $r \succ_{H} s$ for every $i, j=1,2, \ldots, n$ such that $i<j$ and every residents $r$ in $R_{i}$ and $s$ in $R_{j}$.

For each $i, j=1,2, \ldots, n$, we define $R_{i, j}:=R_{i} \cup R_{i+1} \cup \cdots \cup R_{j}$.

Our algorithm SuperM is described as follows.

\section{Algorithm SuperM}

Step 1. Define $i:=1, M_{0}:=\emptyset$, and $D_{0}:=\emptyset$. 
Step 2. If $i \leq n$, then do the following steps. Otherwise, go to Step 3.

(2-a) For each resident $r$ in $R_{i}$, we define $T_{r}$ by

$$
T_{r}:=\left\{e \in E(r) \backslash D_{i-1} \mid \forall f \in E(r) \backslash D_{i-1}: e \succsim_{r} f\right\} .
$$

(2-b) If there exists a resident $r$ in $R_{i}$ such that $\left|T_{r}\right|>1$, then output null and halt (i.e., there exists no super-stable matching in $G$ ).

(2-c) Define $E_{i}:=\cup_{r \in R_{i}} T_{r}$. If $M_{i-1} \cup E_{i} \notin \mathcal{F}$, then output null and halt.

(2-d) Define $M_{i}:=M_{i-1} \cup E_{i}$.

(2-e) Define

$$
L_{i}:=\left\{(r, h) \in E \backslash D_{i-1} \mid r \in R_{i+1, n}, M_{i}+(r, h) \notin \mathcal{F}\right\} .
$$

Furthermore, define $D_{i}:=D_{i-1} \cup L_{i}$.

(2-f) Update $i:=i+1$ and go back to the beginning of Step 2.

Step 3. Output $M_{n}$ and halt (i.e., $M_{n}$ is a super-stable matching in $G$ ).

End of Algorithm

In the sequel, we prove the correctness of the algorithm SuperM.

Lemma 5. Assume that the algorithm SuperM halts when $i=\delta$. Then, for any edge e in $D_{\delta-1}$, there does not exist a super-stable matching $N$ in $G$ such that $e \in N$.

Proof. An edge $e$ in $D_{\delta-1}$ is said to be bad, if there exists a super-stable matching $N$ in $G$ such that $e \in N$. We prove this lemma by contradiction. Assume that there exists a bad edge in $D_{\delta-1}$. Define $\Delta$ as the set of integers $\ell$ in $\{1,2, \ldots, \delta-1\}$ such that there exists a bad edge in $L_{\ell}$. Let $j$ be the minimum integer in $\Delta$. Let $e=(r, h)$ and $N$ be a bad edge in $L_{j}$ and a super-stable matching such that $e \in N$, respectively. Since $e$ belongs to $L_{j}, M_{j}+e \notin \mathcal{F}$. Define $C:=\mathrm{C}_{\mathbf{N}}\left(e, M_{j}\right)$. If $C$ is a subset of $N$, then this contradicts the fact that $N \in \mathcal{F}$. Thus, $C \backslash N$ is not empty. For every edge $(s, p)$ in $C \backslash N$, since $r \in R_{j+1, k}$ and $s \in R_{1, j}$, we have $s \succ_{H} r$.

We first assume that there exists an edge $f=(s, p)$ in $C \backslash N$ such that $N(s) \succ_{s} f$. Since $e$ belongs to $C \cap N$, we have $e \neq f$. This implies that $f$ belongs to $M_{j}$, i.e., $f \in T_{s}$. Thus, the definition of the algorithm SuperM implies that $N(s)$ belongs to $D_{j-1}$, i.e., $N(s) \in L_{k}$ for an integer $k$ in $\{1,2, \ldots, j-1\}$. Furthermore, since $D_{j-1} \subseteq D_{\delta-1}$ and $N$ is super-stable, $N(s)$ is bad. This contradicts the minimality of $j$.

Next we assume that $f \succsim_{s} N(s)$ for every edge $f=(s, p)$ in $C \backslash N$. If there exists an edge $f$ in $C \backslash N$ such that $N+f \in \mathcal{F}$, then this contradicts the fact that $N$ is super-stable. Thus, we assume that $N+f \notin \mathcal{F}$ for every edge $f$ in $C \backslash N$. For each edge $f$ in $C \backslash N$, we define $C_{f}$ as $\mathrm{C}_{\mathbf{N}}(f, N)$. Since $N$ is super-stable, $t \succ_{H} s$ for every edge $f=(s, p)$ in $C \backslash N$ and every edge $g=(t, q)$ in $C_{f}-f$. For every edge $f=(s, p)$ in $C \backslash N$, since $s \succ_{H} r$, this implies that $e \notin C_{f}$. In addition, $f \in C \cap C_{f}$ for every edge $f$ in $C \backslash N$. Thus, Lemma 3 implies that there exists a circuit $C^{\prime}$ of $\mathbf{N}$ such that $C^{\prime}$ is a subset of $\left(C \cup C^{*}\right) \backslash(C \backslash N)$, where $C^{*}$ is $\cup_{f \in C \backslash N} C_{f}$. Thus, since $C_{f}-f$ is a subset of $N, C^{\prime}$ is a subset of $N$. This contradicts the fact that $N \in \mathcal{F}$.

Lemma 6. Assume that the algorithm SuperM halts when $i=\delta$, and we are given a super-stable matching $N$ in $G$. Then, for every resident $r$ in $R_{1, \delta}$ such that $T_{r}=\emptyset$ (resp., $\left.T_{r} \neq \emptyset\right)$, we have $N(r)=\emptyset\left(\right.$ resp., $\left.N(r) \in T_{r}\right)$. 
Proof. For every resident $r$ in $R_{1, \delta}$ such that $T_{r}=\emptyset$, every edge in $E(r)$ belongs to $D_{\delta-1}$. Thus, Lemma 5 implies that $N(r)=\emptyset$ for every resident $r$ in $R_{1, \delta}$ such that $T_{r}=\emptyset$.

From here, we consider a resident $r$ in $R_{1, \delta}$ such that $T_{r} \neq \emptyset$. We assume that $r$ belongs to $R_{j}$ for an integer $j$ in $\{1,2, \ldots, \delta\}$. If $N(r) \in T_{r}$, then the proof is done. Thus, we assume that $N(r)$ does not belong to $T_{r}$, and we prove this lemma by contradiction. If $N(r) \succsim_{r} e$ for an edge $e$ in $T_{r}$ (notice that $f \sim_{r} g$ for every edges $f, g$ in $T_{r}$ ), then the definition of $T_{r}$ implies that $N(r)$ belongs to $D_{\delta-1}$, which contradicts Lemma 5. Thus, we assume that $e \succ_{r} N(r)$ for an edge $e$ in $T_{r}$. In addition, we assume that there exists no resident $s$ in $R_{1, j-1}$ such that $T_{s} \neq \emptyset$ and $e \succ_{s} N(s)$ for an edge $e$ in $T_{s}$. Notice that such a resident $r$ clearly exists.

Let $e$ be an edge in $T_{r}$. Since $e$ belongs to $T_{r}$, $e$ does not belong to $D_{j-1}$. Since this implies that $e \notin D_{j-2}$ and $e \notin L_{j-1}$, we have $M_{j-1}+e \in \mathcal{F}$. Since $N(r)$ does not belong to $T_{r}$, $e$ does not belong to $N$. Furthermore, since $N$ is super-stable, $N+e \notin \mathcal{F}$ and $s \succ_{H} r$ for every edge $(s, p)$ in $\mathrm{C}_{\mathbf{N}}^{-}(e, N)$. Here we prove that $\mathrm{C}_{\mathbf{N}}(e, N)$ is a subset of $M_{j-1}+e$. This contradicts the fact that $M_{j-1}+e \in \mathcal{F}$. Let $f=(s, p)$ be an edge in $\mathrm{C}_{\mathbf{N}}^{-}(e, N)$. Since $s \succ_{H} r, s$ belongs to $R_{k}$ for an integer $k$ in $\{1,2, \ldots, j-1\}$. This and the definition of the algorithm superM imply that $\left|T_{s}\right| \leq 1$. If $T_{s}=\emptyset$, then in the same way as above we can prove that $N(s)=\emptyset$, which contradict $f=N(s)$. Thus, $\left|T_{s}\right|=1$. In this case, the unique edge in $T_{s}$ belongs to $M_{j-1}$. Thus, if $f \in T_{s}$, then the proof is done. Assume that $f$ does not belong to $T_{s}$. In this case, since $f=N(s)$, the definition of $r$ implies that $N(s) \succsim_{s} g$ for an edge $g$ in $T_{s}$. This implies that $f$ belongs to $D_{\delta-1}$. Since $f$ belongs to a super-stable matching $N$, this contradicts Lemma 5 .

Lemma 7. If the algorithm SuperM outputs $M_{n}$, then $M_{n}$ is a super-stable matching in $G$.

Proof. For every $\ell=1,2, \ldots, n$ and every resident $r$ in $R_{\ell},\left|E_{\ell}(r)\right| \leq 1$. Thus, since $M_{\ell} \in \mathcal{F}$ for every $\ell=1,2, \ldots, n, M_{n}$ is a matching in $G$. What remains is to prove that $M_{n}$ is super-stable. Let $e=(r, h)$ be an edge in $E \backslash M_{n}$. We prove that at least one of $r$ and $H$ does not weakly prefer $e$ on $M_{n}$. If $M_{n}(r) \succ_{r} e$, then the proof is done. Thus, we consider the case where $e \succsim_{r} M_{n}(r)$. Assume that $r$ belongs to $R_{j}$ for an integer $j$ in $\{1,2, \ldots, n\}$. Since $M_{n}(r)$ belongs to $T_{r}$ and $\left|T_{r}\right|=1$, the definition of the algorithm SuperM implies that $e$ belongs to $L_{k}$ for an integer $k$ in $\{1,2 \ldots, j-1\}$. Thus, $M_{k}+e \notin \mathcal{F}$ and $s \succ_{H} r$ for every edge $(s, p)$ in $\mathrm{C}_{\mathbf{N}}^{-}\left(e, M_{k}\right)$. Since $M_{k}$ is a subset of $M_{n}$, Lemma 2 implies that $M_{n}+e \notin \mathcal{F}$ and $\mathrm{C}_{\mathbf{N}}\left(e, M_{k}\right)=\mathrm{C}_{\mathbf{N}}\left(e, M_{n}\right)$. Thus, $s \succ_{H} r$ for every edge $f=(s, p)$ in $\mathrm{C}_{\mathbf{N}}^{-}\left(e, M_{n}\right)$. This completes the proof.

Lemma 8. If the algorithm SuperM outputs null, then there exists no super-stable matching in $G$.

Proof. We assume that the algorithm SuperM outputs null when $i=\delta$. We prove this lemma by contradiction. Assume that there exists a super-stable matching $N$ in $G$.

We first consider the case where in Step (2-b) the algorithm SuperM outputs null. In this case, there exists a resident $r$ in $R_{\delta}$ such that $\left|T_{r}\right|>1$. Lemma 6 implies that $N(r)$ belongs to $T_{r}$. Let $e$ be an edge in $T_{r}$ such that $e \neq N(r)$. Since $e$ and $N(r)$ belong to $T_{r}$, we have $e \sim_{r} N(r)$. Thus, what remains is to prove that $N+e \in \mathcal{F}$ and/or there exists an edge $f=(s, p)$ in $N$ such that $N+e-f \in \mathcal{F}$ and $r \succsim_{H} s$, which contradicts the fact that $N$ is super-stable. If $N+e \in \mathcal{F}$, then proof is done. Thus, we assume that $N+e \notin \mathcal{F}$ and $s \succ_{H} r$ (i.e., $s$ belongs to $R_{1, \delta-1}$ ) for every edge $(s, p)$ in $\mathrm{C}_{\mathbf{N}}^{-}(e, N)$. Since $e \in T_{r}, M_{\delta-1}+e \in \mathcal{F}$. Lemma 6 and the definition of the algorithm SuperM imply that $N(s)=M_{\delta-1}(s)$ for every resident $s$ in $R_{1, \delta-1}$. These imply that $\mathrm{C}_{\mathbf{N}}(e, N)$ is a subset of $M_{\delta-1}+e$, which contradicts the fact that $M_{\delta-1}+e \in \mathcal{F}$.

Next we consider the case where in Step (2-c) the algorithm SuperM outputs null. That is, $M_{\delta-1} \cup E_{\delta} \notin \mathcal{F}$. Lemma 6 and the definition of the algorithm of SuperM imply that $M_{\delta-1}(r)=$ 
$N(r)$ for every resident $r$ in $R_{1, \delta-1}$ and $E_{\delta}(r)=N(r)$ for every resident $r$ in $R_{\delta}$. These imply that $M_{\delta-1} \cup E_{\delta}$ is a subset of $N$, which contradicts the fact that $N \in \mathcal{F}$.

Lemmas 7 and 8 imply the following theorem.

Theorem 9. The algorithm SuperM can decide whether there exists a super-stable matching in $G$, and find such a matching, if one exists.

Here we consider the time complexity of SuperM. Define $m:=|E|$, and we denote by $\gamma$ the time required to decide whether $F \in \mathcal{F}$ for one subset $F$ of $E$. For simplicity, we assume that $\gamma=\Omega(m)$ and $m \geq|R|$. Since the number of iterations of Step 2 is at most $m$, we consider the time complexity of one iteration of Step 2. Clearly, Step (2-e) is the bottleneck and the time complexity of this step is $O(m \gamma)$. Thus, the time complexity of the algorithm SuperM is $O\left(m^{2} \gamma\right)$.

\section{Strongly Stable Matchings}

In this section, we give an algorithm for the problem of deciding whether there exists a strongly stable matching in $G$, and find such a matching, if one exists. We define subsets $R_{1}, R_{2}, \ldots, R_{n}$ of $R$ and the notation $R_{i, j}$ in the same way as in Section 3.

Our algorithm StrongM is described as follows.

\section{Algorithm StrongM}

Step 1. Define $i:=1, M_{0}:=\emptyset, D_{0}:=\emptyset$, and $P_{0}:=\emptyset$.

Step 2. If $i \leq n$, then do the following steps. Otherwise, go to Step 3.

(2-a) For each resident $r$ in $R_{i}$, we define $T_{r}$ by

$$
T_{r}:=\left\{e \in E(r) \backslash D_{i-1} \mid \forall f \in E(r) \backslash D_{i-1}: e \succsim_{r} f\right\} .
$$

(2-b) Define

$$
E_{i}:=\bigcup_{r \in R_{i}} T_{r}, \quad P_{i}:=\bigcup_{\ell=1,2, \ldots, i} E_{\ell}, \quad \mathbf{N}_{i}:=\left(\mathbf{N} \mid P_{i}\right) / P_{i-1}, \quad R_{i}^{*}:=\left\{a \in R_{i} \mid T_{r} \neq \emptyset\right\} .
$$

(2-c) If $r_{\mathbf{N}_{i}}\left(E_{i}\right)>\left|R_{i}^{*}\right|$, then output null and halt.

(2-d) Define

$$
\mathcal{U}_{i}:=\left\{F \subseteq E_{i}\left|\forall r \in R_{i}:\right| F(r) \mid \leq 1\right\}, \quad \mathbf{A}_{i}:=\left(E_{i}, \mathcal{U}_{i}\right) .
$$

Notice that $\mathbf{A}_{i}$ is a matroid. Then, find a maximum-size common independent set $F_{i}$ of $\mathbf{A}_{i}$ and $\mathbf{N}_{i}$.

(2-e) If $\left|F_{i}\right|<\left|R_{i}^{*}\right|$, then output null and halt. Otherwise, define $M_{i}:=M_{i-1} \cup F_{i}$.

(2-f) Define

$$
L_{i}:=\left\{(r, h) \in E \backslash D_{i-1} \mid r \in R_{i+1, n}, M_{i}+(r, h) \notin \mathcal{F}\right\} .
$$

Furthermore, define $D_{i}:=D_{i-1} \cup L_{i}$.

(2-g) Update $i:=i+1$ and go to the beginning of Step 2 . 
Step 3. Output $M_{n}$ and halt.

\section{End of Algorithm}

In the sequel, we prove the correctness of the algorithm StrongM.

Lemma 10. Assume that the algorithm StrongM halts when $i=\delta$. Then,

1. $F_{\ell}$ is a base of $\mathbf{N}_{\ell}$ for every $\ell=1,2, \ldots, \delta-1$, and

2. $M_{\ell}$ is a base of $\mathbf{N} \mid P_{\ell}$ for every $\ell=1,2, \ldots, \delta-1$.

Proof. We first consider the statement (1). Let us fix an integer $\ell$ in $\{1,2, \ldots, \delta-1\}$. Since the algorithm StrongM does not output null when $i=\ell$, we have $r_{\mathbf{N}_{\ell}}\left(E_{\ell}\right) \leq\left|R_{\ell}^{*}\right|$ and $\left|F_{\ell}\right| \geq\left|R_{\ell}^{*}\right|$. These imply that $r_{\mathbf{N}_{i}}\left(E_{\ell}\right) \leq\left|F_{\ell}\right|$. Furthermore, since $F_{\ell}$ is an independent set of $\mathbf{N}_{\ell}$, we have $\left|F_{\ell}\right| \leq r_{\mathbf{N}_{\ell}}\left(E_{\ell}\right)$. These imply that $\left|F_{\ell}\right|=r_{\mathbf{N}_{\ell}}\left(E_{\ell}\right)$, i.e., $F_{\ell}$ is a base of $\mathbf{N}_{\ell}$.

Next we prove the statement (2) by induction on $\ell$. The statement (2) for $\ell=1$ is equivalent to the statement (1) for $\ell=1$. Let $\xi$ be an integer in $\{1,2, \ldots, \delta-2\}$. Assume that the statement (2) holds for the case of $\ell=\xi$, and we consider the case of $\ell=\xi+1$. Since $\mathbf{N}\left|P_{\xi}=\left(\mathbf{N} \mid P_{\xi+1}\right)\right| P_{\xi}$ and $F_{\xi+1}$ is a base of $\mathbf{N}_{\xi+1}=\left(\mathbf{N} \mid P_{\xi+1}\right) / P_{\xi}$, the induction assumption and Lemma 4(2) imply that $M_{\xi+1}$ is a base of $\mathbf{N} \mid P_{\xi+1}$.

Lemma 11. Assume that the algorithm StrongM halts when $i=\delta$. Then, for any edge $e$ in $D_{\delta-1}$, there does not exists a strongly stable matching $N$ in $G$ such that $e \in N$.

Proof. We say that an edge $e$ in $D_{\delta-1}$ is bad, if there exists a strongly stable matching $N$ in $G$ such that $e \in N$. We prove this lemma by contradiction. That is, we assume that there exists a bad edge in $D_{\delta-1}$. Define $\Delta$ as the set of integers $\ell$ in $\{1,2, \ldots, \delta-1\}$ such that there exists a bad edge in $L_{\ell}$. Let $j$ be the minimum integer in $\Delta$. Furthermore, let $e=(r, h)$ and $N$ be a bad edge in $L_{j}$ and a strongly stable matching such that $e \in N$, respectively. Since $e$ belongs to $L_{j}, M_{j}+e \notin \mathcal{F}$. Define $C:=\mathrm{C}_{\mathbf{N}}\left(e, M_{j}\right)$ (notice that $M_{j} \in \mathcal{F}$ follows from Lemma 10(2)). If $C$ is a subset of $N$, then this contradicts the fact that $N \in \mathcal{F}$. Thus, $C \backslash N$ is not empty. For every edge $(s, p)$ in $C \backslash N$, since $r \in R_{j+1, k}$ and $s \in R_{1, j}$, we have $s \succ_{H} r$.

We first assume that there exists an edge $f=(s, p)$ in $C \backslash N$ such that $N(s) \succ_{s} f$. Since $e$ belongs to $N, e \neq f$. This implies that $f$ belongs to $M_{j}$, i.e., $f \in T_{s}$. Thus, the definition of the algorithm StrongM implies that $N(s)$ belongs to $D_{j-1}$, i.e., $N(s) \in L_{k}$ for an integer $k$ in $\{1,2, \ldots, j-1\}$. Furthermore, since $D_{j-1} \subseteq D_{\delta-1}$ and $N$ is strongly stable, $N(s)$ is bad, which contradicts the minimality of $j$.

Next we assume that $f \succsim_{s} N(s)$ for every edge $f=(s, p)$ in $C \backslash N$. If there exists an edge $f$ in $C \backslash N$ such that $N+f \in \mathcal{F}$, then this contradicts the fact that $N$ is strongly stable. Thus, we assume that $N+f \notin \mathcal{F}$ for every edge $f$ in $C \backslash N$. For each edge $f$ in $C \backslash N$, we define $C_{f}$ as $\mathrm{C}_{\mathbf{N}}(f, N)$. Since $N$ is strongly stable, $t \succsim_{H} s$ for every edge $f=(s, p)$ in $C \backslash N$ and every edge $g=(t, q)$ in $C_{f}-f$. For every edge $f=(s, p)$ in $C \backslash N$, since $s \succ_{H} r$, this implies that $e \notin C_{f}$. In addition, $f \in C \cap C_{f}$ for every edge $f$ in $C \backslash N$. Thus, Lemma 3 implies that there exists a circuit $C^{\prime}$ of $\mathbf{N}$ such that $C^{\prime}$ is a subset of $N$. This contradicts the fact that $N \in \mathcal{F}$.

Lemma 12. Assume that the algorithm StrongM halts when $i=\delta$, and we are given a strongly stable matching $N$ in $G$. Then, for every resident $r$ in $R_{1, \delta}$ such that $T_{r}=\emptyset\left(\right.$ resp., $\left.T_{r} \neq \emptyset\right)$, we have $N(r)=\emptyset\left(\right.$ resp., $\left.N(r) \in T_{r}\right)$. 
Proof. For every resident $r$ in $R_{1, \delta}$ such that $T_{r}=\emptyset$, every edge in $E(r)$ belongs to $D_{\delta-1}$. Thus, Lemma 11 implies that $N(r)=\emptyset$ for every resident $r$ in $R_{1, \delta}$ such that $T_{r}=\emptyset$.

From here, we consider a resident $r$ in $R_{1, \delta}$ such that $T_{r} \neq \emptyset$. We assume that $r$ belongs to $R_{j}$ for an integer $j$ in $\{1,2, \ldots, \delta\}$. If $N(r) \in T_{r}$, then the proof is done. Thus, we assume that $N(r)$ does not belong to $T_{r}$, and we prove this lemma by contradiction. If $N(r) \succsim_{r} e$ for an edge $e$ in $T_{r}$, then the definition of $T_{r}$ implies that $N(r)$ belongs to $D_{\delta-1}$, which contradicts Lemma 11. Thus, we assume that $e \succ_{r} N(r)$ for an edge $e$ in $T_{r}$. Furthermore, we assume that there exists no resident $s$ in $R_{1, j-1}$ such that $T_{s} \neq \emptyset$ and $e \succ_{s} N(s)$ for an edge $e$ in $T_{s}$. Notice that such a resident $r$ clearly exists.

Let $e$ be an edge in $T_{r}$. Since $e$ belongs to $T_{r}, M_{j-1}+e \in \mathcal{F}$. Since $N(r)$ does not belong to $T_{r}, e$ does not belong to $N$. In addition, since $N$ is strongly stable, $N+e \notin \mathcal{F}$ and $s \succ_{H} r$ for every edge $(s, p)$ in $\mathrm{C}_{\mathbf{N}}^{-}(e, N)$. Define $C:=\mathrm{C}_{\mathbf{N}}(e, N)$. Since $M_{j-1}+e \in \mathcal{F}, C$ is not a subset of $M_{j-1}+e$. Let $f=(s, p)$ be an edge in $C \backslash\left(M_{j-1}+e\right)$. Since $s \succ_{H} r, s \in R_{k}$ for an integer $k$ in $\{1,2, \ldots, j-1\}$. In the same way as above, we can prove that $f \in T_{s}$ or $g \succ_{s} f$ for an edge $g$ in $T_{s}$. The assumption of $r$ implies that $f$ belongs to $T_{s}$, i.e., $f \in E_{k}$. Since $f \notin\left(M_{j-1}+e\right)$ implies that $f$ does not belong to $F_{k}$ and Lemma 10(1) implies that $F_{k}$ is a base of $\mathbf{N}_{k}, F_{k}+f$ is not an independent set of $\mathbf{N}_{k}=\left(\mathbf{N} \mid P_{k}\right) / P_{k-1}$. Thus, since Lemma 10(2) implies that $M_{k-1}$ is a base of $\left(\mathbf{N} \mid P_{k}\right) \mid P_{k-1}$, Lemma 4(3) implies that $M_{k}+f \notin \mathcal{F}$. Since $M_{k} \subseteq M_{j-1}, M_{j-1}+f \notin \mathcal{F}$. For each edge $f$ in $C \backslash\left(M_{j-1}+e\right)$, we define $C_{f}:=\mathrm{C}_{\mathbf{N}}\left(f, M_{j-1}\right)$. For every edge $f$ in $C \backslash\left(M_{j-1}+e\right)$, since $e \notin M_{j-1}$ and $f \neq e, e$ does not belong to $C_{f}$. Furthermore, $f \in C \cap C_{f}$ for every edge $f$ in $C \backslash\left(M_{j-1}+e\right)$. Thus, Lemma 3 implies that there exists a circuit $C^{\prime}$ of $\mathbf{N}$ that $C^{\prime}$ is a subset of $M_{j-1}+e$, which contradicts the fact that $M_{j-1}+e \in \mathcal{F}$.

Lemma 13. Assume that the algorithm StrongM halts when $i=\delta$, and we are given a strongly stable matching $M$. Then, for every $\ell=1,2, \ldots, \delta$, the following statements hold.

1. If $\ell>1$, then $N \cap P_{\ell-1}$ is a base of $\mathbf{N} \mid P_{\ell-1}$.

2. $N \cap E_{\ell}$ is a base of $\mathbf{N}_{\ell}$.

Proof. We prove this lemma by induction on $\ell$. We first consider the case of $\ell=1$, and we prove this case by contradiction. Assume that $N \cap E_{1}$ is not a base of $\mathbf{N}_{1}=\mathbf{N} \mid E_{1}$. Then, since $N \in \mathcal{F}$, $N \cap E_{1}$ is an independent set of $\mathbf{N}_{1}$. Since $N \cap E_{1}$ is not a base of $\mathbf{N}_{1}$, there exists an edge $e=(r, h)$ in $E_{1} \backslash N$ such that $\left(N \cap E_{1}\right)+e \in \mathcal{F}$. Since $D_{0}=\emptyset$ and $e \in T_{r}$, we have $e \succsim_{r} N(r)$. Thus, if $N+e \in \mathcal{F}$, then this contradicts the fact that $N$ is strongly stable. Thus, we assume that $N+e \notin \mathcal{F}$. Then, since $\left(N \cap E_{1}\right)+e \in \mathcal{F}, \mathrm{C}_{\mathbf{N}}(e, N)$ is not a subset of $\left(N \cap E_{1}\right)+e$. Let $f=(s, p)$ be an edge in $C_{\mathbf{N}}(e, N) \backslash\left(\left(N \cap E_{1}\right)+e\right)$. If $s \in R_{j}$ for an integer $j$ in $\{2,3, \ldots, n\}$, then $r \succ_{H} s$. This contradicts the fact that $N$ is strongly stable. Thus, we have $s \in R_{1}$. Since $f \in E(s)$ (i.e., $E(s) \neq \emptyset$ ) and $D_{0}=\emptyset$, we have $T_{s} \neq \emptyset$. Thus, since $f \in N$, Lemma 12 implies that $f \in T_{s}$, i.e., $f \in E_{1}$. This contradicts the fact that $f \notin N \cap E_{1}$.

Let $\xi$ be an integer in $\{1,2, \ldots, \delta-2\}$, and assume that this lemma holds for the case of $\ell=\xi$, and we consider the case of $\ell=\xi+1$. We first prove the statement (1). If $\xi=1$, then the statement (1) for $\ell=\xi+1$ is equivalent to the statement (2) for the case of $\ell=1$. Assume that $\xi \geq 2$. The induction assumption implies that $N \cap P_{\xi-1}$ is a base of $\left(\mathbf{N} \mid P_{\xi}\right) \mid P_{\xi-1}$ and $N \cap E_{\xi}$ is a base of $\left(\mathbf{N} \mid P_{\xi}\right) / P_{\xi-1}$. Thus, Lemma 4(2) implies that $N \cap P_{\xi}$ is a base of $\mathbf{N} \mid P_{\xi}$, which completes the proof. Next we prove the statement (2) by contradiction. Assume that $N \cap E_{\xi+1}$ is not a base of $\mathbf{N}_{\xi+1}$. Then, there exists an edge $e=(r, h)$ in $E_{\xi+1} \backslash N$ such that $\left(N \cap E_{\xi+1}\right)+e$ is an independent set of $\mathbf{N}_{\xi+1}=\left(\mathbf{N} \mid P_{\xi+1}\right) / P_{\xi}$. Since the statement (1) for the case of $\ell=\xi+1$ implies that $N \cap P_{\xi}$ is a base of $\left(\mathbf{N} \mid P_{\xi+1}\right) \mid P_{\xi}$, Lemma 4(1) implies that $\left(N \cap P_{\xi+1}\right)+e \in \mathcal{F}$. Since $e$ belongs to $E_{\xi+1}$ (i.e., $T_{r}$ ), Lemma 12 implies that $e \sim_{r} N(r)$. Thus, if $N+e \in \mathcal{F}$, then 
this contradicts the fact that $N$ is strongly stable. Thus, we consider the case where $N+e \notin \mathcal{F}$. Since $\left(N \cap P_{\xi+1}\right)+e \in \mathcal{F}, \mathrm{C}_{\mathbf{N}}(e, N)$ is not a subset of $\left(N \cap P_{\xi+1}\right)+e$. Let $f=(s, p)$ be an edge in $\mathrm{C}_{\mathbf{N}}(e, N) \backslash\left(\left(N \cap P_{\xi+1}\right)+e\right)$. Since $f=N(s)$, Lemma 12 implies that $f$ belongs to $T_{s}$. This and $f \notin P_{\xi+1}$ imply that $s \in R_{j}$ for an integer $j$ in $\{\xi+2, \xi+3, \ldots, n\}$. Thus, $r \succ_{H} s$, which contradicts the fact that $N$ is strongly stable.

Lemma 14. If the algorithm StrongM outputs $M_{n}$, then $M_{n}$ is a strongly stable matching in $G$.

Proof. Since Lemma 10(2) implies that $M_{n} \in \mathcal{F}$ and $\left|M_{n}(r)\right| \leq 1$ clearly holds for every resident $r$ in $R, M_{n}$ is a matching in $G$. What remains is to prove that $M_{n}$ is strongly stable.

Let $e=(r, h)$ be an edge in $E \backslash M_{n}$. If $M_{n}(r) \succ_{r} e$, then $r$ does not weakly prefer $e$ on $M$. Thus, we do not need to consider this case. Assume that $e \succsim_{r} M_{n}(r)$ and $r \in R_{j}$ for an integer $j$ in $\{1,2, \ldots, n\}$. We first consider the case where $e \succ_{r} M_{n}(r)$. In this case, since $M_{n}(r) \in T_{r}$, the definition of the algorithm StrongM implies that $e \in L_{k}$ for an integer $k$ in $\{1,2, \ldots, j-1\}$. Thus, $M_{k}+e \notin \mathcal{F}$ and $s \succ_{H} r$ for every edge $(s, p)$ in $\mathrm{C}_{\mathbf{N}}^{-}\left(e, M_{k}\right)$. Since $M_{k} \subseteq M_{n}$, Lemma 2 implies that $\mathrm{C}_{\mathbf{N}}\left(e, M_{k}\right)=\mathrm{C}_{\mathbf{N}}\left(e, M_{n}\right)$. Thus, $s \succ_{H} r$ for every edge $f=(s, p)$ in $\mathrm{C}_{\mathbf{N}}^{-}\left(e, M_{n}\right)$. This implies that $H$ does not weakly prefer $e$ on $M$. Lastly we consider the case where $e \sim_{r} M_{n}(r)$. We first assume that $e \notin T_{r}$. In this case, the definition of the algorithm StrongM implies that $e \in L_{k}$ for an integer $k$ in $\{1,2, \ldots, j-1\}$. Thus, we can treat this case in the same way as above. Next we assume that $e \in T_{r}$. Notice that $e \in E_{j} \backslash F_{j}$. Since Lemma 10(1) implies that $F_{j}$ is a base of $\mathbf{N}_{j}, F_{j}+e$ is not an independent set of $\left(\mathbf{N} \mid P_{j}\right) / P_{j-1}$. In addition, Lemma 10(2) implies that $M_{j-1}$ is a base of $\left(\mathbf{N} \mid P_{j}\right) \mid P_{j-1}$. Thus, Lemma 4(3) implies that $M_{j}+e \notin \mathcal{F}$. Since $M_{j} \subseteq P_{j}$, we have $s \succsim_{H} r$ for each edge $(s, p)$ in $\mathrm{C}_{\mathbf{N}}\left(e, M_{j}\right)$. Since $M_{j} \subseteq M_{n}$, Lemma 2 implies that $M_{n}+e \notin \mathcal{F}$ and $\mathrm{C}_{\mathbf{N}}\left(e, M_{j}\right)=\mathrm{C}_{\mathbf{N}}\left(e, M_{n}\right)$. Thus, we have $s \succsim_{H} r$ for every edge $f=(s, p)$ in $\mathrm{C}_{\mathbf{N}}^{-}\left(e, M_{n}\right)$. This completes the proof.

Lemma 15. If the algorithm StrongM outputs null, then there exists no strongly stable matching in $G$.

Proof. We assume that the algorithm StrongM outputs null when $i=\delta$. We prove this lemma by contradiction. Assume that there exists a strongly stable matching $N$ in $G$.

We first consider the case where in Step (2-c) the algorithm StrongM outputs null. In this case, we have $r_{\mathbf{N}_{\delta}}\left(E_{\delta}\right)>\left|R_{\delta}^{*}\right|$. Since Lemma 13(2) implies that $N \cap E_{\delta}$ is a base of $\mathbf{N}_{\delta}$, we have $\left|N \cap E_{\delta}\right|=r_{\mathbf{N}_{\delta}}\left(E_{\delta}\right)$, which implies that $\left|N \cap E_{\delta}\right|>\left|R_{\delta}^{*}\right|$. However, since $N$ is a matching and $E_{\delta}$ is a subset of $\cup_{r \in R_{\delta}^{*}} E(r),\left|N \cap E_{\delta}\right| \leq\left|R_{\delta}^{*}\right|$, which contradicts the fact that $\left|N \cap E_{\delta}\right|>\left|R_{\delta}^{*}\right|$.

Next we consider the case where in Step (2-e) the algorithm StrongM outputs null. In this case, we have $\left|F_{\delta}\right|<\left|R_{\delta}^{*}\right|$. Since $N$ is a matching in $G,|N(r)| \leq 1$ for every resident $r$ in $R_{\delta}$. Furthermore, Lemma 13(2) implies that $N \cap E_{\delta}$ is an independent set of $\mathbf{N}_{\delta}$. Thus, $N \cap E_{\delta}$ is a common independent set of $\mathbf{A}_{\delta}$ and $\mathbf{N}_{\delta}$. Since $F_{\delta}$ is a maximum-size common independent set of $\mathbf{A}_{\delta}$ and $\mathbf{N}_{\delta}$, we have $\left|N \cap E_{\delta}\right| \leq\left|F_{\delta}\right|<\left|R_{\delta}^{*}\right|$. This implies that there exists a resident $r$ in $R_{\delta}^{*}$ such that $N(r) \notin E_{\delta}$. However, since $T_{r} \subseteq E_{\delta}$, this contradicts Lemma 12 .

Lemmas 14 and 15 imply the following theorem.

Theorem 16. The algorithm StrongM can decide whether there exists a strongly stable matching in $G$, and find such a matching, if one exists.

Here we consider the time complexity of StrongM. Define $m:=|E|$, and we denote by $\gamma$ the time required to decide whether $F \in \mathcal{F}$ for one subset $F$ of $E$. For simplicity, we assume that $\gamma=\Omega(m)$ and $m \geq|R|$. Since the number of iterations of Step $\mathbf{2}$ is at most $m$, we consider the time complexity of one iteration of Step 2. Clearly, Step (2-d) is the bottleneck. Lemma 4(1) 
implies that by computing bases of $\mathbf{N} \mid P_{\ell}$ for all $\ell=1,2, \ldots, n$ in $O\left(m^{2} \gamma\right)$ time, we can decide in $O(\gamma)$ time whether $F$ is an independent set of $\mathbf{N}_{\ell}$ for an integer $\ell$ in $\{1,2, \ldots, n\}$ and one subset $F$ of $E_{\ell}$. Thus, the time complexity of this step is $O\left(m^{2.5} \gamma\right)$, and the time complexity of SuperM is $O\left(m^{3.5} \gamma\right)$.

\section{$5 \quad$ Examples}

In this section, we give examples of concrete models that our model can express.

\subsection{Hospitals/residents setting}

Assume that for each hospital $h$ in $H$, we are given a positive integer $q_{h}$. Then, we define

$$
\mathcal{F}:=\left\{F \subseteq E|\forall h \in H:| F(h) \mid \leq q_{h}\right\} .
$$

It is not difficult to see that $\mathbf{N}=(E, \mathcal{F})$ is a matroid. When we define the matroid $\mathbf{N}$ as above, a subset $M$ of $E$ is a matching if and only if

1. $|M(r)| \leq 1$ for every resident $r$ in $R$, and

2. $|M(h)| \leq q_{h}$ for every hospital $h$ in $H$.

Furthermore, for each matching $M$ in $G$ and each edge $e=(r, h)$ in $E \backslash M, H$ weakly (strictly) prefers $e$ on $M$ if and only if

1. $|M(h)|<q_{h}$, and/or

2. there exists an edge $f=(s, h)$ in $M(h)$ such that $r \succsim_{H} s$ (resp., $r \succ_{H} s$ ).

Thus, our problem is equivalent to the hospitals/residents problem with ties and master lists [12].

\subsection{Student-project allocation setting}

Assume that $H$ is partitioned into non-empty subsets $H_{1}, H_{2}, \ldots, H_{k}$, and we are given positive integers $c_{h}$ and $d_{i}$ for each hospital $h$ in $H$ and each $i=1,2, \ldots, k$, respectively. Then, we define

$$
\begin{aligned}
\mathcal{F}:=\{F \subseteq E \mid & \forall h \in H:|F(h)| \leq c_{h}, \\
& \left.\forall i=1,2, \ldots, k: \sum_{h \in H_{i}}|F(h)| \leq d_{i}\right\} .
\end{aligned}
$$

It is not difficult to see that $\mathbf{N}=(E, \mathcal{F})$ is a matroid. When we define the matroid $\mathbf{N}$ as above, a subset $M$ of $E$ is a matching if and only if

1. $|M(r)| \leq 1$ for every resident $r$ in $R$,

2. $|M(h)| \leq c_{h}$ for every hospital $h$ in $H$, and

3. $\sum_{h \in H_{i}}|M(h)| \leq d_{i}$ for every $i=1,2, \ldots, k$.

These constraints are exactly the same as the capacity constraints of the student-project allocation problem [1]. For each matching $M$ in $G$ and each edge $e=(r, h)$ in $E \backslash M$ such that $h \in H_{i}$ for an integer $i$ in $\{1,2, \ldots, k\}, H$ weakly (strictly) prefers $e$ on $M$ if and only if at least one of the following conditions is satisfied. 
1. $|M(h)|<c_{h}$ and $\sum_{h \in H_{i}}|M(h)|<d_{i}$.

2. $|M(h)|<c_{h}$ and there exists an edge $f=(s, p)$ in $M$ such that $p \in H_{i}$ and $r \succsim_{H} s$ (resp., $\left.r \succ_{H} s\right)$.

3. There exists an edge $f=(s, h)$ in $M(h)$ such that $r \succsim_{H} s$ (resp., $r \succ_{H} s$ ).

It should be noted that in the above definition, if $|M(h)|<c_{h}$ and $M(r) \in H_{i}$, then $H$ weakly prefers $e$ on $M$ (i.e., the condition (2) is satisfied), but it does not mean that $H$ strictly prefers $e$ on $M$ since $r \nsucc_{H} r$.

\section{References}

[1] D. J. Abraham, R. W. Irving, and D. Manlove. Two algorithms for the student-project allocation problem. Journal of Discrete Algorithms, 5(1):73-90, 2007.

[2] W. H. Cunningham. Improved bounds for matroid partition and intersection algorithms. SIAM Journal on Computing, 15(4):948-957, 1986.

[3] T. Fleiner. A fixed-point approach to stable matchings and some applications. Mathematics of Operations Research, 28(1):103-126, 2003.

[4] D. Gale and L. S. Shapley. College admissions and the stability of marriage. The American Mathematical Monthly, 69(1):9-15, 1962.

[5] R. W. Irving. Stable marriage and indifference. Discrete Applied Mathematics, 48(3):261$272,1994$.

[6] R. W. Irving, D. F. Manlove, and S. Scott. The hospitals/residents problem with ties. In Proceedings of the 7th Scandinavian Workshop on Algorithm Theory, volume 1851 of Lecture Notes in Computer Science, pages 259-271, 2000.

[7] R. W. Irving, D. F. Manlove, and S. Scott. Strong stability in the hospitals/residents problem. In Proceedings of the 20th Annual Symposium on Theoretical Aspects of Computer Science, volume 2607 of Lecture Notes in Computer Science, pages 439-450, 2003.

[8] R. W. Irving, D. F. Manlove, and S. Scott. The stable marriage problem with master preference lists. Discrete Applied Mathematics, 156(15):2959-2977, 2008.

[9] T. Kavitha, K. Mehlhorn, D. Michail, and K. Paluch. Strongly stable matchings in time $O(n m)$ and extension to the hospitals-residents problem. ACM Transactions on Algorithms, 3(2):Article 15, 2007.

[10] D. F. Manlove. Stable marriage with ties and unacceptable partners. Technical Report TR-1999-29, The University of Glasgow, Department of Computing Science, 1999.

[11] D. F. Manlove. Algorithmics of matching under preferences. World Scientific, 2013.

[12] G. O'Malley. Algorithmic aspects of stable matching problems. PhD thesis, The University of Glasgow, 2007.

[13] J. G. Oxley. Matroid theory. Oxford University Press, 2nd edition, 2011. 


\section{List of MI Preprint Series, Kyushu University \\ The Global COE Program \\ Math-for-Industry Education \& Research Hub}

MI

MI2008-1 Takahiro ITO, Shuichi INOKUCHI \& Yoshihiro MIZOGUCHI

Abstract collision systems simulated by cellular automata

MI2008-2 Eiji ONODERA

The intial value problem for a third-order dispersive flow into compact almost Hermitian manifolds

MI2008-3 Hiroaki KIDO

On isosceles sets in the 4-dimensional Euclidean space

MI2008-4 Hirofumi NOTSU

Numerical computations of cavity flow problems by a pressure stabilized characteristiccurve finite element scheme

MI2008-5 Yoshiyasu OZEKI

Torsion points of abelian varieties with values in nfinite extensions over a p-adic field

MI2008-6 Yoshiyuki TOMIYAMA

Lifting Galois representations over arbitrary number fields

MI2008-7 Takehiro HIROTSU \& Setsuo TANIGUCHI

The random walk model revisited

MI2008-8 Silvia GANDY, Masaaki KANNO, Hirokazu ANAI \& Kazuhiro YOKOYAMA

Optimizing a particular real root of a polynomial by a special cylindrical algebraic decomposition

MI2008-9 Kazufumi KIMOTO, Sho MATSUMOTO \& Masato WAKAYAMA

Alpha-determinant cyclic modules and Jacobi polynomials

MI2008-10 Sangyeol LEE \& Hiroki MASUDA

Jarque-Bera Normality Test for the Driving Lévy Process of a Discretely Observed Univariate SDE

MI2008-11 Hiroyuki CHIHARA \& Eiji ONODERA

A third order dispersive flow for closed curves into almost Hermitian manifolds

MI2008-12 Takehiko KINOSHITA, Kouji HASHIMOTO and Mitsuhiro T. NAKAO

On the $L^{2}$ a priori error estimates to the finite element solution of elliptic problems with singular adjoint operator

MI2008-13 Jacques FARAUT and Masato WAKAYAMA

Hermitian symmetric spaces of tube type and multivariate Meixner-Pollaczek polynomials 
MI2008-14 Takashi NAKAMURA

Riemann zeta-values, Euler polynomials and the best constant of Sobolev inequality

MI2008-15 Takashi NAKAMURA

Some topics related to Hurwitz-Lerch zeta functions

MI2009-1 Yasuhide FUKUMOTO

Global time evolution of viscous vortex rings

MI2009-2 Hidetoshi MATSUI \& Sadanori KONISHI

Regularized functional regression modeling for functional response and predictors

MI2009-3 Hidetoshi MATSUI \& Sadanori KONISHI

Variable selection for functional regression model via the $L_{1}$ regularization

MI2009-4 Shuichi KAWANO \& Sadanori KONISHI

Nonlinear logistic discrimination via regularized Gaussian basis expansions

MI2009-5 Toshiro HIRANOUCHI \& Yuichiro TAGUCHII

Flat modules and Groebner bases over truncated discrete valuation rings

MI2009-6 Kenji KAJIWARA \& Yasuhiro OHTA

Bilinearization and Casorati determinant solutions to non-autonomous $1+1$ dimensional discrete soliton equations

MI2009-7 Yoshiyuki KAGEI

Asymptotic behavior of solutions of the compressible Navier-Stokes equation around the plane Couette flow

MI2009-8 Shohei TATEISHI, Hidetoshi MATSUI \& Sadanori KONISHI

Nonlinear regression modeling via the lasso-type regularization

MI2009-9 Takeshi TAKAISHI \& Masato KIMURA

Phase field model for mode III crack growth in two dimensional elasticity

MI2009-10 Shingo SAITO

Generalisation of Mack's formula for claims reserving with arbitrary exponents for the variance assumption

MI2009-11 Kenji KAJIWARA, Masanobu KANEKO, Atsushi NOBE \& Teruhisa TSUDA Ultradiscretization of a solvable two-dimensional chaotic map associated with the Hesse cubic curve

MI2009-12 Tetsu MASUDA

Hypergeometric $\tau$-functions of the q-Painlevé system of type $E_{8}^{(1)}$

MI2009-13 Hidenao IWANE, Hitoshi YANAMI, Hirokazu ANAI \& Kazuhiro YOKOYAMA A Practical Implementation of a Symbolic-Numeric Cylindrical Algebraic Decomposition for Quantifier Elimination

MI2009-14 Yasunori MAEKAWA

On Gaussian decay estimates of solutions to some linear elliptic equations and its applications 
MI2009-15 Yuya ISHIHARA \& Yoshiyuki KAGEI

Large time behavior of the semigroup on $L^{p}$ spaces associated with the linearized compressible Navier-Stokes equation in a cylindrical domain

MI2009-16 Chikashi ARITA, Atsuo KUNIBA, Kazumitsu SAKAI \& Tsuyoshi SAWABE

Spectrum in multi-species asymmetric simple exclusion process on a ring

MI2009-17 Masato WAKAYAMA \& Keitaro YAMAMOTO

Non-linear algebraic differential equations satisfied by certain family of elliptic functions

MI2009-18 Me Me NAING \& Yasuhide FUKUMOTO

Local Instability of an Elliptical Flow Subjected to a Coriolis Force

MI2009-19 Mitsunori KAYANO \& Sadanori KONISHI

Sparse functional principal component analysis via regularized basis expansions and its application

MI2009-20 Shuichi KAWANO \& Sadanori KONISHI

Semi-supervised logistic discrimination via regularized Gaussian basis expansions

MI2009-21 Hiroshi YOSHIDA, Yoshihiro MIWA \& Masanobu KANEKO

Elliptic curves and Fibonacci numbers arising from Lindenmayer system with symbolic computations

MI2009-22 Eiji ONODERA

A remark on the global existence of a third order dispersive flow into locally Hermitian symmetric spaces

MI2009-23 Stjepan LUGOMER \& Yasuhide FUKUMOTO

Generation of ribbons, helicoids and complex scherk surface in laser-matter Interactions

MI2009-24 Yu KAWAKAMI

Recent progress in value distribution of the hyperbolic Gauss map

MI2009-25 Takehiko KINOSHITA \& Mitsuhiro T. NAKAO

On very accurate enclosure of the optimal constant in the a priori error estimates for $H_{0}^{2}$-projection

MI2009-26 Manabu YOSHIDA

Ramification of local fields and Fontaine's property (Pm)

MI2009-27 Yu KAWAKAMI

Value distribution of the hyperbolic Gauss maps for flat fronts in hyperbolic threespace

MI2009-28 Masahisa TABATA

Numerical simulation of fluid movement in an hourglass by an energy-stable finite element scheme

MI2009-29 Yoshiyuki KAGEI \& Yasunori MAEKAWA

Asymptotic behaviors of solutions to evolution equations in the presence of translation and scaling invariance 
MI2009-30 Yoshiyuki KAGEI \& Yasunori MAEKAWA

On asymptotic behaviors of solutions to parabolic systems modelling chemotaxis

MI2009-31 Masato WAKAYAMA \& Yoshinori YAMASAKI

Hecke's zeros and higher depth determinants

MI2009-32 Olivier PIRONNEAU \& Masahisa TABATA

Stability and convergence of a Galerkin-characteristics finite element scheme of lumped mass type

MI2009-33 Chikashi ARITA

Queueing process with excluded-volume effect

MI2009-34 Kenji KAJIWARA, Nobutaka NAKAZONO \& Teruhisa TSUDA

Projective reduction of the discrete Painlevé system of type $\left(A_{2}+A_{1}\right)^{(1)}$

MI2009-35 Yosuke MIZUYAMA, Takamasa SHINDE, Masahisa TABATA \& Daisuke TAGAMI Finite element computation for scattering problems of micro-hologram using DtN map

MI2009-36 Reiichiro KAWAI \& Hiroki MASUDA

Exact simulation of finite variation tempered stable Ornstein-Uhlenbeck processes

MI2009-37 Hiroki MASUDA

On statistical aspects in calibrating a geometric skewed stable asset price model

MI2010-1 Hiroki MASUDA

Approximate self-weighted LAD estimation of discretely observed ergodic OrnsteinUhlenbeck processes

MI2010-2 Reiichiro KAWAI \& Hiroki MASUDA

Infinite variation tempered stable Ornstein-Uhlenbeck processes with discrete observations

MI2010-3 Kei HIROSE, Shuichi KAWANO, Daisuke MIIKE \& Sadanori KONISHI

Hyper-parameter selection in Bayesian structural equation models

MI2010-4 Nobuyuki IKEDA \& Setsuo TANIGUCHI

The Itô-Nisio theorem, quadratic Wiener functionals, and 1-solitons

MI2010-5 Shohei TATEISHI \& Sadanori KONISHI

Nonlinear regression modeling and detecting change point via the relevance vector machine

MI2010-6 Shuichi KAWANO, Toshihiro MISUMI \& Sadanori KONISHI

Semi-supervised logistic discrimination via graph-based regularization

MI2010-7 Teruhisa TSUDA

UC hierarchy and monodromy preserving deformation

MI2010-8 Takahiro ITO

Abstract collision systems on groups 
MI2010-9 Hiroshi YOSHIDA, Kinji KIMURA, Naoki YOSHIDA, Junko TANAKA \& Yoshihiro MIWA

An algebraic approach to underdetermined experiments

MI2010-10 Kei HIROSE \& Sadanori KONISHI

Variable selection via the grouped weighted lasso for factor analysis models

MI2010-11 Katsusuke NABESHIMA \& Hiroshi YOSHIDA

Derivation of specific conditions with Comprehensive Groebner Systems

MI2010-12 Yoshiyuki KAGEI, Yu NAGAFUCHI \& Takeshi SUDOU

Decay estimates on solutions of the linearized compressible Navier-Stokes equation around a Poiseuille type flow

MI2010-13 Reiichiro KAWAI \& Hiroki MASUDA

On simulation of tempered stable random variates

MI2010-14 Yoshiyasu OZEKI

Non-existence of certain Galois representations with a uniform tame inertia weight

MI2010-15 Me Me NAING \& Yasuhide FUKUMOTO

Local Instability of a Rotating Flow Driven by Precession of Arbitrary Frequency

MI2010-16 Yu KAWAKAMI \& Daisuke NAKAJO

The value distribution of the Gauss map of improper affine spheres

MI2010-17 Kazunori YASUTAKE

On the classification of rank 2 almost Fano bundles on projective space

MI2010-18 Toshimitsu TAKAESU

Scaling limits for the system of semi-relativistic particles coupled to a scalar bose field

MI2010-19 Reiichiro KAWAI \& Hiroki MASUDA

Local asymptotic normality for normal inverse Gaussian Lévy processes with highfrequency sampling

MI2010-20 Yasuhide FUKUMOTO, Makoto HIROTA \& Youichi MIE

Lagrangian approach to weakly nonlinear stability of an elliptical flow

MI2010-21 Hiroki MASUDA

Approximate quadratic estimating function for discretely observed Lévy driven SDEs with application to a noise normality test

MI2010-22 Toshimitsu TAKAESU

A Generalized Scaling Limit and its Application to the Semi-Relativistic Particles System Coupled to a Bose Field with Removing Ultraviolet Cutoffs

MI2010-23 Takahiro ITO, Mitsuhiko FUJIO, Shuichi INOKUCHI \& Yoshihiro MIZOGUCHI Composition, union and division of cellular automata on groups

MI2010-24 Toshimitsu TAKAESU

A Hardy's Uncertainty Principle Lemma in Weak Commutation Relations of HeisenbergLie Algebra 
MI2010-25 Toshimitsu TAKAESU

On the Essential Self-Adjointness of Anti-Commutative Operators

MI2010-26 Reiichiro KAWAI \& Hiroki MASUDA

On the local asymptotic behavior of the likelihood function for Meixner Lévy processes under high-frequency sampling

MI2010-27 Chikashi ARITA \& Daichi YANAGISAWA

Exclusive Queueing Process with Discrete Time

MI2010-28 Jun-ichi INOGUCHI, Kenji KAJIWARA, Nozomu MATSUURA \& Yasuhiro OHTA Motion and Bäcklund transformations of discrete plane curves

MI2010-29 Takanori YASUDA, Masaya YASUDA, Takeshi SHIMOYAMA \& Jun KOGURE On the Number of the Pairing-friendly Curves

MI2010-30 Chikashi ARITA \& Kohei MOTEGI

Spin-spin correlation functions of the $q$-VBS state of an integer spin model

MI2010-31 Shohei TATEISHI \& Sadanori KONISHI

Nonlinear regression modeling and spike detection via Gaussian basis expansions

MI2010-32 Nobutaka NAKAZONO

Hypergeometric $\tau$ functions of the $q$-Painlevé systems of type $\left(A_{2}+A_{1}\right)^{(1)}$

MI2010-33 Yoshiyuki KAGEI

Global existence of solutions to the compressible Navier-Stokes equation around parallel flows

MI2010-34 Nobushige KUROKAWA, Masato WAKAYAMA \& Yoshinori YAMASAKI

Milnor-Selberg zeta functions and zeta regularizations

MI2010-35 Kissani PERERA \& Yoshihiro MIZOGUCHI

Laplacian energy of directed graphs and minimizing maximum outdegree algorithms

MI2010-36 Takanori YASUDA

CAP representations of inner forms of $S p(4)$ with respect to Klingen parabolic subgroup

MI2010-37 Chikashi ARITA \& Andreas SCHADSCHNEIDER

Dynamical analysis of the exclusive queueing process

MI2011-1 Yasuhide FUKUMOTO\& Alexander B. SAMOKHIN

Singular electromagnetic modes in an anisotropic medium

MI2011-2 Hiroki KONDO, Shingo SAITO \& Setsuo TANIGUCHI

Asymptotic tail dependence of the normal copula

MI2011-3 Takehiro HIROTSU, Hiroki KONDO, Shingo SAITO, Takuya SATO, Tatsushi TANAKA \& Setsuo TANIGUCHI

Anderson-Darling test and the Malliavin calculus

MI2011-4 Hiroshi INOUE, Shohei TATEISHI \& Sadanori KONISHI

Nonlinear regression modeling via Compressed Sensing 
MI2011-5 Hiroshi INOUE

Implications in Compressed Sensing and the Restricted Isometry Property

MI2011-6 Daeju KIM \& Sadanori KONISHI

Predictive information criterion for nonlinear regression model based on basis expansion methods

MI2011-7 Shohei TATEISHI, Chiaki KINJYO \& Sadanori KONISHI

Group variable selection via relevance vector machine

MI2011-8 Jan BREZINA \& Yoshiyuki KAGEI

Decay properties of solutions to the linearized compressible Navier-Stokes equation around time-periodic parallel flow

Group variable selection via relevance vector machine

MI2011-9 Chikashi ARITA, Arvind AYYER, Kirone MALLICK \& Sylvain PROLHAC

Recursive structures in the multispecies TASEP

MI2011-10 Kazunori YASUTAKE

On projective space bundle with nef normalized tautological line bundle

MI2011-11 Hisashi ANDO, Mike HAY, Kenji KAJIWARA \& Tetsu MASUDA

An explicit formula for the discrete power function associated with circle patterns of Schramm type

MI2011-12 Yoshiyuki KAGEI

Asymptotic behavior of solutions to the compressible Navier-Stokes equation around a parallel flow

MI2011-13 Vladimír CHALUPECKÝ \& Adrian MUNTEAN

Semi-discrete finite difference multiscale scheme for a concrete corrosion model: approximation estimates and convergence

MI2011-14 Jun-ichi INOGUCHI, Kenji KAJIWARA, Nozomu MATSUURA \& Yasuhiro OHTA Explicit solutions to the semi-discrete modified KdV equation and motion of discrete plane curves

MI2011-15 Hiroshi INOUE

A generalization of restricted isometry property and applications to compressed sensing

MI2011-16 Yu KAWAKAMI

A ramification theorem for the ratio of canonical forms of flat surfaces in hyperbolic three-space

MI2011-17 Naoyuki KAMIYAMA

Matroid intersection with priority constraints

MI2012-1 Kazufumi KIMOTO \& Masato WAKAYAMA

Spectrum of non-commutative harmonic oscillators and residual modular forms

MI2012-2 Hiroki MASUDA

Mighty convergence of the Gaussian quasi-likelihood random fields for ergodic Levy driven SDE observed at high frequency 
MI2012-3 Hiroshi INOUE

A Weak RIP of theory of compressed sensing and LASSO

MI2012-4 Yasuhide FUKUMOTO \& Youich MIE

Hamiltonian bifurcation theory for a rotating flow subject to elliptic straining field

MI2012-5 Yu KAWAKAMI

On the maximal number of exceptional values of Gauss maps for various classes of surfaces

MI2012-6 Marcio GAMEIRO, Yasuaki HIRAOKA, Shunsuke IZUMI, Miroslav KRAMAR, Konstantin MISCHAIKOW \& Vidit NANDA

Topological Measurement of Protein Compressibility via Persistence Diagrams

MI2012-7 Nobutaka NAKAZONO \& Seiji NISHIOKA

Solutions to a $q$-analog of Painlevé III equation of type $D_{7}^{(1)}$

MI2012-8 Naoyuki KAMIYAMA

A new approach to the Pareto stable matching problem

MI2012-9 Jan BREZINA \& Yoshiyuki KAGEI

Spectral properties of the linearized compressible Navier-Stokes equation around time-periodic parallel flow

MI2012-10 Jan BREZINA

Asymptotic behavior of solutions to the compressible Navier-Stokes equation around a time-periodic parallel flow

MI2012-11 Daeju KIM, Shuichi KAWANO \& Yoshiyuki NINOMIYA

Adaptive basis expansion via the extended fused lasso

MI2012-12 Masato WAKAYAMA

On simplicity of the lowest eigenvalue of non-commutative harmonic oscillators

MI2012-13 Masatoshi OKITA

On the convergence rates for the compressible

Navier- Stokes equations with potential force

MI2013-1 Abuduwaili PAERHATI \& Yasuhide FUKUMOTO

A Counter-example to Thomson-Tait-Chetayev's Theorem

MI2013-2 Yasuhide FUKUMOTO \& Hirofumi SAKUMA

A unified view of topological invariants of barotropic and baroclinic fluids and their application to formal stability analysis of three-dimensional ideal gas flows

MI2013-3 Hiroki MASUDA

Asymptotics for functionals of self-normalized residuals of discretely observed stochastic processes

MI2013-4 Naoyuki KAMIYAMA

On Counting Output Patterns of Logic Circuits

MI2013-5 Hiroshi INOUE

RIPless Theory for Compressed Sensing 
MI2013-6 Hiroshi INOUE

Improved bounds on Restricted isometry for compressed sensing

MI2013-7 Hidetoshi MATSUI

Variable and boundary selection for functional data via multiclass logistic regression modeling

MI2013-8 Hidetoshi MATSUI

Variable selection for varying coefficient models with the sparse regularization

MI2013-9 Naoyuki KAMIYAMA

Packing Arborescences in Acyclic Temporal Networks

MI2013-10 Masato WAKAYAMA

Equivalence between the eigenvalue problem of non-commutative harmonic oscillators and existence of holomorphic solutions of Heun's differential equations, eigenstates degeneration, and Rabi's model

MI2013-11 Masatoshi OKITA

Optimal decay rate for strong solutions in critical spaces to the compressible Navier-

Stokes equations

MI2013-12 Shuichi KAWANO, Ibuki HOSHINA, Kazuki MATSUDA \& Sadanori KONISHI

Predictive model selection criteria for Bayesian lasso

MI2013-13 Hayato CHIBA

The First Painleve Equation on the Weighted Projective Space

MI2013-14 Hidetoshi MATSUI

Variable selection for functional linear models with functional predictors and a functional response

MI2013-15 Naoyuki KAMIYAMA

The Fault-Tolerant Facility Location Problem with Submodular Penalties

MI2013-16 Hidetoshi MATSUI

Selection of classification boundaries using the logistic regression

MI2014-1 Naoyuki KAMIYAMA

Popular Matchings under Matroid Constraints

MI2014-2 Yasuhide FUKUMOTO \& Youichi MIE

Lagrangian approach to weakly nonlinear interaction of Kelvin waves and a symmetrybreaking bifurcation of a rotating flow

MI2014-3 Reika AOYAMA

Decay estimates on solutions of the linearized compressible Navier-Stokes equation around a Parallel flow in a cylindrical domain

MI2014-4 Naoyuki KAMIYAMA

The Popular Condensation Problem under Matroid Constraints 
MI2014-5 Yoshiyuki KAGEI \& Kazuyuki TSUDA

Existence and stability of time periodic solution to the compressible Navier-Stokes equation for time periodic external force with symmetry

MI2014-6 This paper was withdrawn by the authors.

MI2014-7 Masatoshi OKITA

On decay estimate of strong solutions in critical spaces for the compressible NavierStokes equations

MI2014-8 Rong ZOU \& Yasuhide FUKUMOTO

Local stability analysis of azimuthal magnetorotational instability of ideal MHD flows

MI2014-9 Yoshiyuki KAGEI \& Naoki MAKIO

Spectral properties of the linearized semigroup of the compressible Navier-Stokes equation on a periodic layer

MI2014-10 Kazuyuki TSUDA

On the existence and stability of time periodic solution to the compressible NavierStokes equation on the whole space

MI2014-11 Yoshiyuki KAGEI \& Takaaki NISHIDA

Instability of plane Poiseuille flow in viscous compressible gas

MI2014-12 Chien-Chung HUANG, Naonori KAKIMURA \& Naoyuki KAMIYAMA

Exact and approximation algorithms for weighted matroid intersection

MI2014-13 Yusuke SHIMIZU

Moment convergence of regularized least-squares estimator for linear regression model

MI2015-1 Hidetoshi MATSUI

Sparse regularization for multivariate linear models for functional data

MI2015-2 Reika AOYAMA \& Yoshiyuki KAGEI

Spectral properties of the semigroup for the linearized compressible Navier-Stokes equation around a parallel flow in a cylindrical domain

MI2015-3 Naoyuki KAMIYAMA

Stable Matchings with Ties, Master Preference Lists, and Matroid Constraints 